Pacific Journal of Mathematics

TWISTED COHOMOLOGY AND ENUMERATION OF VECTOR 


\title{
TWISTED COHOMOLOGY AND ENUMERATION OF VECTOR BUNDLES
}

\author{
LAWRENCE L. LARMORE
}

\begin{abstract}
In the present paper we give a technique for completely enumerating real 4-plane bundles over a 4-dimensional space, real 5-plane bundles over a 5-dimensional space, and real 6plane bundles over a 6-dimensional space. We give a complete table of real and complex vector bundles over real projective space $P_{k}$, for $k \leqq 5$. Some interesting results are:

(0.1.1.) Over $P_{5}$, there are four oriented 4-plane bundles which could be the normal bundle to an immersion of $P^{5}$ in $R^{9}$, i.e., have stable class $2 h+2$, where $h$ is the canonical line bundle. Of these, two have a unique complex structure.

(0.1.2.) Over $P_{5}$ there is an oriented 4-plane bundle which we call $C$, which has stable class $6 h-2$, which has two distinct complex structures. $D$, the conjugate of $C$, i.e., reversed orientation, has no complex structure.

(0.1.3) Over $P_{5}$, there are no 4-plane bundles of stable class $5 h-1$ or $7 h-3$.
\end{abstract}

0.2. In reading the tables (4.5.2) and (4.6), remember that if $\xi$ : $P_{k} \rightarrow B O(n)$ or $\xi: P_{k} \rightarrow B U(n)$ is a locally oriented (i.e., oriented over base-point) real or complex vector bundle, and if

$$
a \in H^{k}\left(P_{k} ; \pi_{k}(B O(n), \xi)\right)
$$

(local coefficients if $\xi$ unoriented) or $a \in H^{k}\left(P_{k} ; \pi_{k}(B U(n))\right.$, then $\xi+a$ is a vector bundle obtained by cutting out a disk in the top cell of $P_{k}$ and joining a sphere with some vector bundle on it.

0.3. Since some of the homotopy groups of $B O(n)$ are acted upon nontrivially by $Z_{2} \cong \pi_{1}(B O(n))$ for $n$ even, we study cohomology with local coefficients in $\S 3$.

1.2. From here on, we assume that all spaces are connected C. W.-complexes with base-point, all maps are b.p.p. (base-pointpreserving) and that all homotopies are b.p.p.

For any space $Y$, we choose a Postnikov system for $Y$, that is: for each integer $n \geqq 0$, a space $(Y)_{n}$ and a map $P_{n}: Y \rightarrow(Y)_{n}$ which induces an isomorphism in homotopy through dimension $n$, where all homotopy groups of $(Y)_{n}$ are zero above $n$; for each $n \geqq 1$ a fibration $p_{n}:(Y)_{n} \rightarrow(Y)_{n-1}$ such that $p_{n} P_{n}=P_{n-1}$. The fiber of each $p_{n}$ is then an Eilenberg-MacLane space of type $\left(\pi_{n}(Y), n\right)$. If $X$ is a space of finite dimension $m$, then $[X ; Y]$, the set of homotopy classes of maps 
from $X$ to $Y$, is in one-to-one correspondence with $\left[X ;(Y)_{m}\right]$.

Definition (1.2.1). For any integer $n \geqq 1$, let $G_{n}(Y)$ be the sheaf over $(Y)_{1}$ whose stalk over every $y$ is defined to be $\pi_{n}\left(p^{-1} y\right)$, which is isomorphic to $\pi_{n}(Y)$ (where $\left.p=p_{2} \cdots p_{n}:(Y)_{n} \rightarrow(Y)_{1}\right)$ if $n \geqq 2$; $\pi_{1}\left((Y)_{1}, y\right)$ if $n=1$. If $X$ is any space and $f: X \rightarrow(Y)_{1}$ is a map, let $\pi_{n}(Y, f)$ be the sheaf $f^{-1} G_{n}(Y)$ over $X$. This sheaf depends only on the homotopy class of $f$. If $g: X \rightarrow(Y)_{m}$ is a map for any integer $m \geqq 1$, or if $h: X \rightarrow Y$ is a map, let $\pi_{n}(Y, g)$ denote $\pi_{n}\left(Y, p_{2} \cdots p_{m} g\right)$ and let $\pi_{n}(Y, n)$ denote $\pi_{n}\left(Y, P_{1} h\right)$.

Definition (1.2.2). If $f$ and $g$ are maps from $X$ to $(Y)_{n}$ for any $n \geqq 2$, which agree on $A$, and if $F: X \times I \rightarrow(Y)_{n-1}$ is a homotopy of $p_{n} f$ with $p_{n} g$ which holds $A$ fixed, let $\delta^{n}(f, g ; F) \in H^{n}\left(X, A ; \pi_{n}(Y, f)\right)$ be the obstruction to lifting $F$ to a homotopy of $f$ with $g$ which holds $A$ fixed.

REMARK (1.2.3). If $g: X \rightarrow(Y)_{n}$ is another map which agrees with $f$ on $A$, and if $G$ is a homotopy of $p_{n} g$ with $p_{n} h$ which holds $A$ fixed, then $\delta^{n}(f, g ; F)+\delta^{n}(g, h ; G)=\delta^{n}(f, h ; F+G)$, where, for each $(x, t) \in X \times I$,

$$
(F+G)(x, t)=\left\{\begin{array}{l}
F(x, 2 t) \quad \text { if } \quad 0 \leqq t \leqq \frac{1}{2} \\
G(x, 2 t-1) \quad \text { if } \quad \frac{1}{2} \leqq t \leqq 1 .
\end{array}\right.
$$

Definition (1.2.4). Let $X$ be a space, let $A \subset X$ be any subcomplex (possible empty), let $f: X \rightarrow(Y)_{n}$ be a map for some integer $n \geqq 2$, and let $a$ be an element of $H^{n}\left(X, A ; \pi_{n}(Y, f)\right)$. We define $f+a$ to be that map from $X$ to $(Y)_{n}$, unique up to fiber homotopy with $A$ held fixed, such that $p_{n}(f+a)=p_{n} f$ and $\delta^{n}(f, f+a)=a$, where $C$ is the constant homotopy.

REMARK (1.2.5). If $b$ is any other element of $H^{n}\left(X, A ; \pi_{n}(Y, f)\right)$, then $f+(a+b)=(f+a)+b$.

REMARK (1.2.6). If $g:\left(X^{\prime}, A^{\prime}\right) \rightarrow(X, A)$ is a map, where $\left(X^{\prime} A^{\prime}\right)$ is any other C. W. pair, then $(f+a) g=g f+g^{*} a$.

MaIn Theorem (1.2.7). For any $a \in H^{n}\left(X, A ; \pi_{n}(Y, f)\right), f+a$ is homotophic to $f$, rel $A$, if and only if $\delta^{n}(f, f ; F)=a$ for some homotopy $F$ of $p_{n} f$ with itself which holds $A$ fixed.

Proof. Let $C$ be the constant homotopy of $p_{n} f$ with itself. On the one hand, if $F$ is any homotopy of $p_{n} f$ with itself which holds 
$A$ fixed, let $a=\delta^{n}(f, f ; F)$. Then $\delta^{n}(f+a, f ; F)=\delta^{n}(f+a, f ; C)+$ $\delta^{n}(f, f ; F)=-a+a=0$. Thus $F$ may be lifted to a homotopy of $f+a$ with $f$. On the other hand, if $G$ is a homotopy of $f+a$ with $f$, then $\delta^{n}\left(f, f ; p_{n} G\right)=\delta^{n}(f, f+a ; C)+\delta^{n}\left(f+a, f ; p_{n} G\right)=a+0=a$.

Definition (1.2.8). Let $L_{f}$ be the subgroup of $H^{n}\left(X, A ; \pi_{n}(Y, f)\right)$ consisting of all $a$ such that $f+a$ is homotopic to $f \operatorname{rel} A$. Then the set of all homotopy $\left(\mathrm{rel} A\right.$ ) classes of liftings of $p_{n} f$ to $(Y)_{n}$ which agree with $f$ on $A$ is in a one-to-one correspondence with the quotient group $H^{n}\left(X, A ; \pi_{n}(Y, f)\right) / L_{f}$; each coset $a+L_{f}$ corresponds to $f+a$. If $g: X \rightarrow Y$ is a map such that $p_{n} g=f$, let $L_{g}^{n}=L_{f}$. If $h: X \rightarrow(Y)_{m}$ is a map such that $p_{n+1} \cdots p_{m} h=f$, for $m \geqq n$, let $L_{h}^{n}=L_{f}$.

REMARK (1.2.9). If $a \in H^{n}\left(X, A ; \pi_{n}(Y, f)\right)$, then $L_{f+a}=L_{f}$.

Proof. Let $F$ be any homotopy of $p_{n} f=p_{n}(f+a)$ with itself, and let $C$ be the constant homotopy. Then $\delta^{n}(f+a, f+a ; F)=$ $\delta^{n}(f+a, f ; C)+\delta^{n}(f, f ; F)+\delta^{n}(f, f+a ; C)=-a+\delta^{n}(f, f ; F)+a=$ $\delta^{n}(f, f ; F)$.

1.3. In order to calculate $L_{f}$ in specific cases, such as $X$ a projective space, $A=$ base-point, and $Y=B O(m)$ for some $m$, we use a spectral sequence which has the following properties:

(1.3.1) $\quad{ }^{f} E_{2}^{p, q}=E_{2}^{p, q}=H^{p}\left(X, A ; \pi_{q}(Y, f)\right)$ if $2 \leqq q \leqq n, 1 \leqq p \leqq q+1$.

(1.3.2) $\quad E_{2}^{p, q}=0$ for all other values of $p$ and $q$.

(1.3.3) $\quad d_{r}: E_{r}^{p, q} \rightarrow E_{r}^{p+r, q+r-1}$ for all $r \geqq 2$.

(1.3.4) $\quad E_{\infty}^{n, n}=H^{n}\left(X, A ; \pi_{n}(Y, f)\right) / L_{f}$, which, by (1.2.7) and (1.2.8) can be put into one-to-one correspondence with the set of rel $A$ homotopy classes of maps $X \rightarrow(Y)_{n}$ whose projection to $(Y)_{n-1}$ is rel $A$ homotopic to $p_{n} f$.

Basically, what is happening is as follows (where, for any space $Z$ and any map $g: A \rightarrow Z$, the set of rel $A$ homotopy classes of maps $X \rightarrow Z$ which agree with $g$ on $A$ is denoted " $[X ; Z: g]$ "); consider the function:

$$
\left[X ;(Y)_{n}: f \mid A\right] \stackrel{\left(p_{n}\right)_{\sharp}}{\longrightarrow}\left[X ;(Y)_{n-1}: p_{n} f \mid A\right] \text {. }
$$

Now $\left(p_{n}\right)_{\ddagger}$ is just a function of sets, but $\left(p_{n}\right)_{\sharp}^{-1}\left(p_{n} f\right)$ is an Abelian group with 0 the homotopy class of $f$ itself. This group, $E_{\infty}^{n, n}$ of our spectral sequence, depends on the choice of $f$.

We define our spectral sequence via an exact couple:

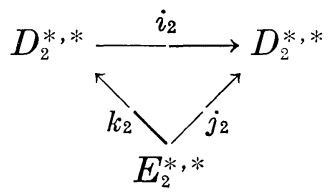


where $E_{2}^{p, q}$ is as defined in (1.3.1) and (1.3.2), where $i_{2}, j_{2}$, and $k_{2}$ have bi-degrees $(-1,-1),(2,1)$, and $(0,0)$ respectively; and where (for all $t \leqq n, M_{t}=$ space of maps from $X$ to $(Y)_{t}$ which agree with $p_{t}^{n} f$ on $A$, compact-open topology):
(1.3.5) $\quad D_{2}^{p, q}=\pi_{q-p}\left(M_{q}, p_{q}^{n} f\right)$ if $0 \leqq q \leqq n$, and $p \leqq q$.
(1.3.6) $\quad D_{2}^{p, q}=0$ if $q<p$ or $q<0$.
(1.3.7) $D_{2}^{p, q}=D_{2}^{p-1, q-1}$ if $q>n$.

Note that $D_{2}^{p, q}$ is only a group if $q=p+1$ and only a set if $q=p$. This will not affect our computation, however.

We proceed to define the homomorphisms $i_{2}, j_{2}$ and $k_{2}$.

(1.3.8) If $q>n$, let $i_{2}$ be the identity. If $q \leqq n$, let $i_{2}=\left(p_{q}\right)_{*}$.

(1.3.9) If $p \leqq q$ and $0 \leqq q<n$, any $x \in D_{2}^{p, q}$ represents a map $\mathrm{g}: X \times I^{q-p} \rightarrow(Y)_{q}$, where $g(x, v)=p_{q}^{n} f(x)$ for all $(x, v) \in X \times \partial I^{q-p} \cup A \times$ $I^{q-p}$. Let $j_{2}(x)=\left(s^{q-p}\right)^{-1} \gamma^{q+2}(g)$, where $\quad s^{q-p}: H^{p+2}\left(X, A ; \pi_{q+1}(Y, f)\right) \rightarrow$ $H^{q+2}\left(X \times I^{q-p}, X \times \partial I^{q-p} \cup A \times I^{q-p} ; \pi_{q+1}(Y, g)\right)$ is the $(q-p)$-fold suspension and $\gamma^{q+2}(g)$ is the obstruction to finding a lifting $h: X \times I^{q-p} \rightarrow$ $(Y)_{q+1}$ of $g$ such that $h(x, v)=p_{q+1}^{n} f(x)$ for all $(x, v) \in X \times \partial I^{q+p} \cup A \times I^{q-p}$. (If $p>q$ or $q<0$ or $q \geqq n, j_{2}: D_{2}^{p, q} \rightarrow E_{2}^{p+2, q+1}$ is obviously the zero map, since $E_{2}^{p+2, q+1}=0$.) This obstruction is zero if and only if $g$ can be lifted; it follows immediately that:

(1.3.10) The sequence $D_{2}^{p+1, q+1} \stackrel{i_{2}}{\longrightarrow} D_{2}^{p, q} \stackrel{j_{2}}{\longrightarrow} E^{p+2, q+1}$ is exact.

Furthermore, since every homotopy, $\operatorname{rel} A$, of $p_{n} f$ with itself represents a loop in $M_{n-1}$ :

(1.3.11) $\quad L_{f}$ is the image of $j_{2}: D_{2}^{n-2, n-1} \rightarrow E_{2}^{n, n}$. For any $2 \leqq q \leqq n$, $1 \leqq p \leqq q$, and any $a \in E_{2}^{p, q}$, let

$$
b=s^{q-p} a \in H^{q}\left(X \times I^{q-p}, X \times \partial I^{q-p} \cup A \times I^{q-p} ; \pi_{q}(Y, C)\right),
$$

where $C(x, v)=p_{q}^{n} f(x)$ for every $(x, v) \in X \times I^{q-p}$. Let $k_{2}(a) \in D_{2}^{p, q}$ be that element represented by the map $C+b$ (cf.1.2.2). It follows from (1.2.3) that $k_{2}$ is a homomorphism if $p<q$; if $p=q$ then $D_{2}^{p, q}$ is only a set anyway. (For other values of $p$ and $q, k_{2}=0$.) Since $p_{q}(C+b)=$ $p_{q} C$, and $C$ represents $0 \in D_{2}^{p, q}$ :

(1.3.12) $\operatorname{Im} k_{2} \subset \operatorname{Ker} i_{2}$.

If, on the other hand, a map g: $X \times I^{q-p} \rightarrow(Y)_{q}$ such that $g=C$ on $X \times \partial I^{q-p} \cup A \times I^{q-p}$ is a representative of a given $a \in \operatorname{Ker} i_{2}$, then $p_{q} g$ is homotopic, rel $X \times \partial I^{q-p} \cup A \times I$, to $p_{q} C$ via a homotopy $F$, then $a=k_{2}\left(\left(s^{q-p}\right)^{-1} \delta^{q}(C, g ; F)\right)$. Thus:

(1.3.13) $\operatorname{Ker} i_{2} \subset \operatorname{Im} k_{2}$.

Somewhat more difficult to show is:

(1.3.14) $\operatorname{Ker} k_{2}=\operatorname{Im} j_{2}$ if $p \leqq q$.

Proof. Let $2 \leqq q \leqq n, 1 \leqq p \leqq q$. Let $g(x, v)=p_{q}^{n} f(x) \in(Y)_{q}$ for all $(x, v) \in X \times I^{q-p} ; g$ represents $0 \in D_{2}^{p, q}$. Let $b \in E_{2}^{p, q}$. Then $b \in \operatorname{Ker} k_{2}$ 
if and only if $s^{q-p} b \in L_{g}$ (cf.1.2.7). If $b=j_{2} a$, then $a$ represents $F$, a homotopy, rel $X \times \partial I^{q-p} \cup A \times I^{q-p}$ of $p_{q} q$ with itself, and $s^{q-p} b=$ $\delta^{q}(g, g ; F) \in L_{g}$. If, on the other hand, $s^{q-p} b \in L_{g}$, then $s^{q-p} b=\delta^{q}(g, g ; F)$ for some homotopy $F$, rel $X \times \partial I^{q-p} \cup A \times I^{q-p}$, of $p_{q} g$ with itself; let $a=[F] \in D^{p-2, q-1}$, and $j_{2} a=b$.

1.4. Since only finitely many of the $E_{2}$ terms are nonzero, we obtain $E_{\infty}$ after a finite number of steps. We also have, by straightforward algebra, an exact sequence

$$
0 \longrightarrow E_{\infty} \stackrel{k_{\infty}}{\longrightarrow} D_{\infty} \stackrel{i_{\infty}}{\longrightarrow} D_{\infty} \longrightarrow 0 \text {. }
$$

Consider now the commutative diagram with exact columns:

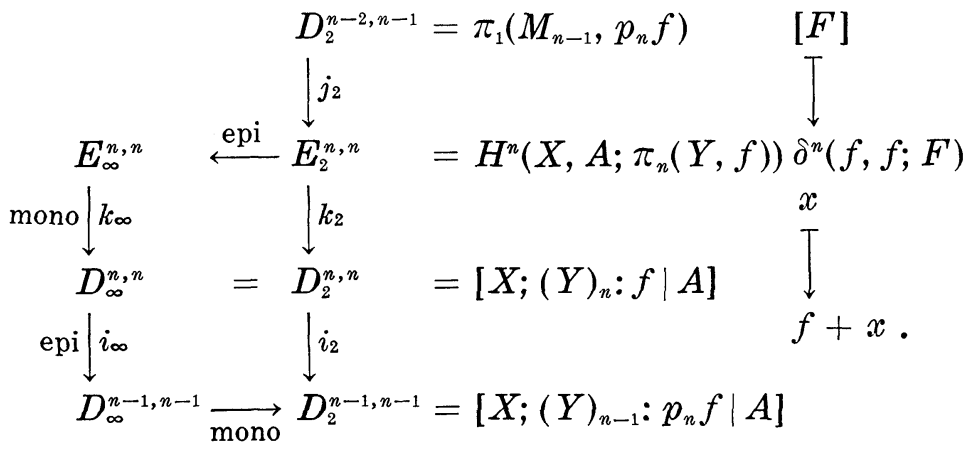

A typical element of $D_{2}^{n-2, n-1}$ is a rel $X \times \partial I \cup A \times I$ homotopy class of homotopies of $p_{n} f$ with itself; if $F$ is such a homotopy, $j_{2}[F]=$ $\delta^{n}(f, f ; F)$, by (1.3.9). If $x \in H^{n}\left(X, A ; \pi_{n}(Y, f)\right), k_{2} x=f+x$, by (1.3.11). Thus $\operatorname{Im} j_{2}=L_{f}$, and $E_{\infty}^{n, n}=H^{n}\left(X, A ; \pi_{n}(Y, f)\right) / L_{f}$, the set of $\operatorname{rel} A$ homotopy classes of liftings of $p_{n} f$.

1.5. If $g:\left(X^{\prime}, A^{\prime}\right) \rightarrow(X, A)$ is a map, $g$ induces a map of spectral sequences.

(1.5.1) $g^{*}:{ }^{f} E_{r}^{p, q} \rightarrow{ }^{f g} E_{r}^{p, q}$ for all $p, q, r$. If $h: Y \rightarrow Z$ is a map, where $Z$ is any other space, $h$ determines a map $h_{m}:(Y)_{m} \rightarrow(Z)_{m}$ for each $m \geqq 0$ [1]. Then $h_{\sharp}: \pi_{1}\left(Y, y_{0}\right) \rightarrow \pi_{1}\left(Z, z_{0}\right)$ induces a sheaf homomorphism from $G_{n}(Y)$ to $\left(h_{1}\right)^{-1} G_{n}(Z)$ which in turn induces a homomorphism.

(1.5.2) $\quad h_{*}: H^{*}\left(X, A ; \pi_{m}(Y, f)\right) \rightarrow H^{*}\left(X, A ; \pi_{m}(Z, h f)\right)$ for all $m \geqq 0$ and a map of spectral sequences

(1.5.3) $h_{*}:{ }^{f} E_{r}^{p, q} \rightarrow{ }^{h f} E_{r}^{p, q}$ for all $p, q, r$.

\section{Nonbase-point-preserving homotopies.}

2.1. Using the techniques of $\S 1$, we can compute all b.p.p. 
homotopy classes of maps from a finite-dimensional space $X$ to a space $Y$. What if we want to know, instead, all free homotopy classes of maps?

2.2. Let $f: X \rightarrow Y$ be any b.p.p. map, and let $a \in \pi_{1}\left(Y, y_{0}\right)$. By the homotopy extension property, we can find a free homotopy $F$ : $X \times I \rightarrow Y$ of $f$ such that $F \mid\left\{x_{0}\right\} \times I$ represents $a$. Let $f^{a}(x)=F(x, 1)$ for any $x \in X ; f^{a}$ is unique up to b.p.p. homotopy, and $f^{a b}\left(f^{a}\right)^{b}$ for any other $b \in \pi_{1}\left(Y, y_{0}\right)$.

THEOREM (2.2.1). If $f$ and $g$ are any b.p.p. maps from $X$ to $Y$, then $f$ is freely homotopic to $g$ if and only if $f^{a}$ is b.p.p. homotopic to $g$ for some $a \in \pi_{1}\left(Y, y_{0}\right)$.

Proof. If $f^{a}$ is b.p.p. homotopic to $g$, then $f$ is obviously freely homotopic to $g$ since $f$ is freely homotopic to $f^{a}$. If, on the other hand, $F: X \times I \rightarrow Y$ is a free homotopy of $f$ with $g$, let $a$ be that element of $\pi_{1}\left(Y, y_{0}\right)$ represented by the loop $F \mid\left\{x_{0}\right\} \times I$. Then $f^{a}=g$ (up to b.p.p. homotopy).

TheOREM (2.2.2). If $n \geqq 2, f: X \rightarrow(Y)_{n}$ is a map,

$$
a \in H^{n}\left(X, x_{0} ; \pi_{n}(Y, f)\right),
$$

and $b \in \pi_{1}\left(Y, y_{0}\right)$, then $(f+a)^{b}=f^{b}+1_{*}^{b}(a)$, where $1_{*}^{b}$ is the homomorphism induced by the map $1^{b}$ (cf.1.5.2), where 1 is the identity map on $(Y)_{n}$.

Proof. The theorem follows from naturality of obstruction theory.

3. Sheaves of local coefficients.

3.1. The homotopy groups of $B O(n)$ are sometimes acted on nontrivially by $\pi_{1}$. We must therefore study twisted sheaves.

Definition (3.1.1). A twisted group is an ordered pair $(G, T), G$ an Abelian group, $T: G \rightarrow G$ an automorphism of order 2. If $X$ is a space, a $(G, T)$-sheaf over $X$ is a fiber bundle over $X$ with fiber $G$ and structural group $Z_{2}$, action determined by $T$. Let $G^{T}[u]$ be the $(G, T)$-sheaf over $P_{\infty}$ obtained by identifying $(x, g)$ with $(T x, T g)$ for all $(x, g) \in S^{\infty} \times G$, where $T: S^{\infty} \rightarrow S^{\infty}$ is the antipodal map.

Definition (3.1.2). If $a \in H^{1}\left(X, x_{0} ; Z_{2}\right)$ and $f:\left(X, x_{0}\right) \rightarrow\left(P_{\infty}, *\right)$ is a map where $f^{*} u=a\left(u=\right.$ fundamental class of $\left.P_{\infty}\right)$, let $G^{T}[\alpha]=$ $f^{-1} G^{T}[u]$. We call $a$ the twisting class of $G^{T}[a]$. 
Proposition (3.1.3). $G^{T}[u]$ is universal in the sense of Steenrod [6], that is, if $G$ is a $(G, T)$-sheaf over a space $X, G \cong G^{T}[a]$ for some unique $a \in H^{1}\left(X, x_{0} ; Z_{2}\right)$.

$$
\text { Proof. } P_{\infty}=B Z_{2} \text {. }
$$

REMARK (3.1.4). If $F: X \times I \rightarrow P_{\infty}$ is a free homotopy of $f$ with itself, where $f^{*} u=a$, then $F$ induces an automorphism of $G^{T}[a] ; 1$ or $T$ depending on whether $F \mid\left\{x_{0}\right\} \times I$ is a trivial loop in $P_{\infty}$ or not.

3.2. If $X$ is a space, $B \subset A \subset X$ are closed, and $S$ is a sheaf over $X$, we have a long exact sequence:

$$
\begin{aligned}
\cdots & \longrightarrow H^{n}(X, A ; S) \longrightarrow H^{n}(X, B ; S) \longrightarrow H^{n}(A, B ; S) \\
& \stackrel{\delta}{\longrightarrow} H^{n+1}(X, A ; S) \longrightarrow \cdots
\end{aligned}
$$

Proposition (3.2.1). If $S$ is a sheaf over a space $X$, and $A \subset X$ is closed, we may find an isomorphism

$$
s: H^{*}(X, A ; S) \longrightarrow H^{*}(X \times I, X \times \partial I \cup A \times I ; S \times I),
$$

called the suspension, of degree 1 , where $S \times I=p^{-1} S ; p: X \times I \rightarrow X$ being the projection.

Proof. Let $S^{\prime}$ be that subsheaf of $S$ such that $S^{\prime} \mid A=0$ and $S^{\prime}|(X-A)=S|(X-A)$. According to Bredon [1],

$$
H^{*}(X, A ; S)=H^{*}\left(X ; S^{\prime}\right)
$$

and

$$
H^{*}(X \times I, X \times \partial I \cup A \times I ; S \times I)=H^{*}\left(X \times I, X \times \partial I ; S^{\prime} \times I\right) .
$$

Now $H^{*}\left(X \times I, X \times\{t\} ; S^{\prime}\right)=0$ for any $t \in I$ [1], and by the long exact sequence of $(X \times I, X \times \partial I, X \times\{1\})$ and excision we have an isomorphism $H^{*}\left(X \times\{0\} ; S^{\prime} \times I\right) \stackrel{\cong}{\longrightarrow} H^{*}\left(X \times I, X \times \partial I ; S^{\prime} \times I\right)$ of degree 1 ; the left group is isomorphic to $H^{*}\left(X ; S^{\prime}\right)$.

3.3. Let $X$ be a space, $A \subset X$ closed. If $\alpha: S \rightarrow S^{\prime}$ is a homomorphism of sheaves over $X$, we get a homomorphism $\alpha_{*}: H^{*}(X, A ; S) \rightarrow$ $H^{*}\left(X, A ; S^{\prime}\right)$. If $S$ and $S^{\prime}$ are sheaves over $X$ and

$$
E: 0 \longrightarrow S \stackrel{i}{\longrightarrow} S^{\prime \prime} \stackrel{p}{\longrightarrow} S^{\prime} \longrightarrow 0
$$

is an extension of $S^{\prime}$ by $S$, then $E$ determines a long exact sequence 


$$
\begin{aligned}
\cdots & \longrightarrow \\
\quad & H^{n}(X, A ; S) \stackrel{i_{*}}{\longrightarrow} H^{n}\left(X, A ; S^{\prime \prime}\right) \stackrel{p_{*}}{\longrightarrow} H^{n}\left(X, A ; S^{\prime}\right) \\
& H^{n+1}(X, A ; S) \longrightarrow \cdots
\end{aligned}
$$

where $\delta^{E}$ is called the Bockstein of $E$.

Proposition (3.3.1). If $S$ and $S^{\prime}$ are sheaves over $X$ and if

$$
E: 0 \longrightarrow S \stackrel{i}{\longrightarrow} S^{\prime \prime} \stackrel{p}{\longrightarrow} S^{\prime} \longrightarrow 0
$$

and

$$
F: 0 \longrightarrow S \stackrel{j}{\longrightarrow} U \stackrel{q}{\longrightarrow} S^{\prime} \longrightarrow 0
$$

are elements of $\operatorname{Ext}\left(S^{\prime}, S\right)$, then $\delta^{E+F}=\delta^{E}+\delta^{F}$.

Proof. We use the Baer sum construction to find

$$
E+F: 0 \longrightarrow S \longrightarrow V \longrightarrow S^{\prime} \longrightarrow 0
$$

our result follows from the commutative diagram, where each row is exact:

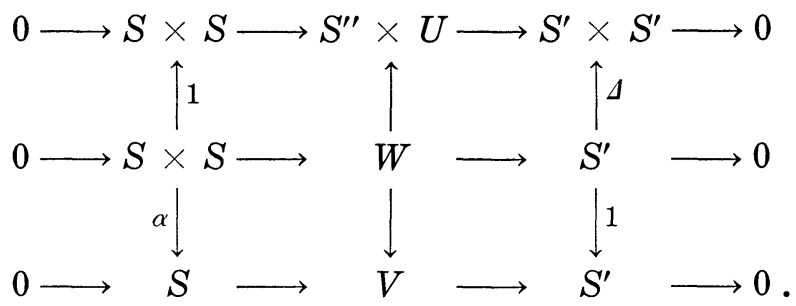

3.4. As Abelian groups $\operatorname{Ext}\left(Z_{2}, Z_{2}\right) \cong Z_{2}$; the nonzero extension is $Z_{4}$. Fix a space $X$; we study Ext of sheaves over $X$.

Proposition 3.4.1. As sheaves over $X$,

$$
\operatorname{Ext}\left(Z_{2}, Z_{2}\right) \cong Z_{2}+H^{1}\left(X, x_{0} ; Z_{2}\right) \text {. }
$$

For any $a \in H^{1}\left(X, x_{0} ; Z_{2}\right),(0, a)$ corresponds to the extension

$$
E_{a}^{0}: 0 \longrightarrow Z_{2} \stackrel{i_{1}}{\longrightarrow}\left(Z_{2}+Z_{2}\right)^{T}[a] \stackrel{p_{2}}{\longrightarrow} Z_{2} \longrightarrow 0,
$$

where $T(x, y)=(x+y, y), i_{1}(x)=(x, 0)$, and $p_{2}(x, y)=y ;(1, a)$ corresponds to

$$
E_{a}^{1}: 0 \longrightarrow Z_{2} \stackrel{m}{\longrightarrow} Z_{4}^{T}[a] \stackrel{e}{\longrightarrow} Z_{2} \longrightarrow 0,
$$

where $T(x)=-x$ for all $x \in Z_{4}, m(1)=2$, and $e(1)=1$.

Proof. Routine computation shows that $E_{a}^{x}+E_{b}^{y}=E_{a+b}^{x+y}$ for any $x, y \in Z_{2}$ and $a, b \in H^{1}\left(X, x_{0} ; Z_{2}\right)$. On the other hand, suppose that 


$$
E: 0 \longrightarrow Z_{2} \stackrel{i}{\longrightarrow} G \stackrel{p}{\longrightarrow} Z_{2} \longrightarrow 0
$$

is some extension. Then the stalk of $G$ at $x_{0}$ is $Z_{4}$, in which case $G=Z_{4}^{T}[a]$ for some $a \in H^{1}\left(X, x_{0} ; Z_{2}\right)$, or it is $Z_{2}+Z_{2}$. In that case, we have an exact sequence of stalks at $x_{0}$ :

$$
0 \longrightarrow Z_{2} \stackrel{i_{1}}{\longrightarrow} Z_{2}+Z_{2} \stackrel{p_{2}}{\longrightarrow} Z_{2} \longrightarrow 0 \text {. }
$$

Since $G$ is locally isomorphic to $Z_{2}+Z_{2}$, it is a fiber bundle with fiber $Z_{2}+Z_{2}$ and structural group Aut $\left(Z_{2}+Z_{2}\right)$. But the only nontrivial automorphism which commutes with $i_{1}: Z_{2} \rightarrow Z_{2}+Z_{2}$ and $p_{2}: Z_{2}+Z_{2} \rightarrow$ $Z_{2}$ is $T$ given above. So the structural group of $G$ may be reduced to $Z_{2} ; G=\left(Z_{2}+Z_{2}\right)^{T}[a]$ for some $a \in H^{1}\left(X, x_{0} ; Z_{2}\right)$. This gives us the isomorphism.

We have the following commutative diagram with both rows exact, for any $a \in H^{1}\left(X, x_{0} ; Z_{2}\right)$ :

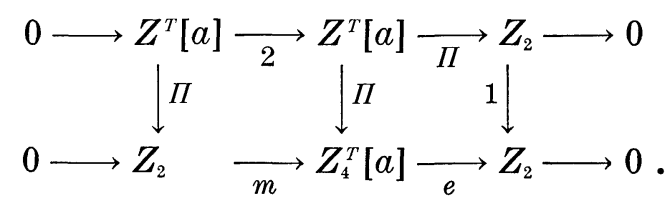

Definition (3.4.2). Let $\beta^{T}[a]$ (or simply $\beta^{T}$, when $a$ is understood) denote the Bockstein of the top row of the above diagram, and let $\left(S_{q}^{1}\right)^{T}[a]$ (or $\left(S_{q}^{1}\right)^{T}$ ) denote the Bockstein of the bottom row.

REMARK (3.4.3). $\Pi_{*} \beta^{T}=\left(S_{q}^{1}\right)^{T}$.

Proposition (3.4.4). For any $n \geqq 0$ and any $x \in H^{n}\left(X, A: Z_{2}\right)$, $\left(S_{q}^{1}\right)^{T} x=S_{q}^{1} x+x \cup a$.

Proof. Samelson [5].

Proposition (3.4.5). For any $n \geqq 0$ and any $x \in H^{n}\left(X, A ; Z_{2}\right)$ $\delta(x)=x \cup a$, where $\delta$ is the Bockstein of $E_{a}^{0}: 0 \rightarrow Z_{2} \rightarrow\left(Z_{2}+Z_{2}\right)^{T}[a] \rightarrow$ $Z_{2} \rightarrow 0$.

Proof. The result follows immediately from (3.3.1), (3.4.1), and (3.4.4).

3.5. Let $T(n, m)=(m-n, m)$ for any $(n, m) \in Z+Z$. If $S$ and $S^{\prime}$ are sheaves over a space $X$, and if $\mu: S \otimes S^{\prime} \rightarrow S^{\prime \prime}$ is a sheaf homomorphism, then we have a cup product defined from

$$
H^{*}(X, A ; S) \otimes H^{*}\left(X, B ; S^{\prime}\right)
$$

to $H^{*}\left(X, A \cup B ; S^{\prime \prime}\right)$ for any closed $A \subset X$ and $B \subset X$. We have thus 
cup products generated by the following relations:

$$
\begin{aligned}
Z^{T}[a] \otimes Z^{T}[b] & =Z^{T}[a+b], Z_{2} \otimes\left(Z_{2}+Z_{2}\right)^{T}[a] \\
& =\left(Z_{2}+Z_{2}\right)^{T}[a], Z \otimes(Z+Z)^{T}[a] \\
& =(Z+Z)^{T}[a], Z^{T}[a] \otimes(Z+Z)^{T}[a]=(Z+Z)^{T}[a]
\end{aligned}
$$

(where $n \otimes(p, q)=(n p, 2 n p-n q)$ ), $Z_{4}^{T}[\alpha] \otimes Z_{4}^{T}[b]=Z_{4}^{T}[a+b]$,

and many others.

Let $(X, A)$ be a C. W.-pair. Let $a \in H^{1}\left(X, x_{0} ; Z_{2}\right)$ and

$$
\alpha=\beta^{T}[a](1) \in H^{1}\left(X ; Z^{T}[a]\right) .
$$

We have the following commutative diagram; where

$$
i_{1} x=(x, 0), T(x, y)=(y-x, y), j_{1} x=(x, 2 x),
$$

and $q_{2}(x, y)=y-2 x$.

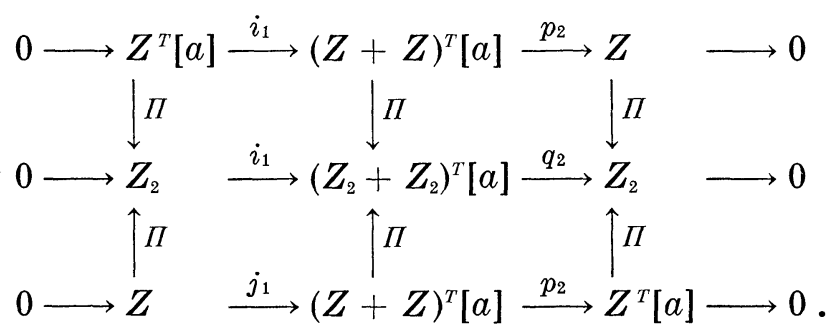

Proposition (3.5.1). The Bockstein homomorphisms $\delta_{1}$ and $\delta_{2}$ are both cup products with $\alpha$.

Proof. By (3.4.3) and (3.4.4) we may compute that

$$
H^{1}\left(P_{\infty} ; Z^{T}[u]\right) \cong Z_{2}
$$

and is generated by $\bar{u}=\beta^{T}(1)$.

Let $x \in H^{n}(X, A ; Z)$. If $n=0$, then the universal example is $X=P_{\infty}, A=\varnothing, x=1$. Then $\alpha=\bar{u}$. Now $H^{0}\left(P_{\infty} ; Z^{T}\right)=0$, so $\left(j_{1}\right)_{*}$ : $H^{0}\left(P_{\infty} ; Z\right) \leftarrow H^{0}\left(P_{\infty} ;(Z+Z)^{T}\right)$ is an isomorphism, and $p_{2} j_{1}=2$. Thus $1 \notin \operatorname{Im}\left(p_{2}\right)_{*}$, so $\delta_{1}(1)=\bar{u}$. If $n \geqq 1$, the universal example is $X=$ $K(Z, n) \times P_{\infty}, A=* \times P_{\infty}, x=v_{n} \times 1$. Then $\alpha=p^{*} \bar{u}$, where $p: X \rightarrow P_{\infty}$ is projection onto the second factor. Now routine computations using (3.4.3) and (3.4.4) show that $H^{n+1}\left(X, A ; Z^{T}\right) \cong Z_{2}$ and is generated by $\left(v_{n} \times 1\right) \cup p^{*} \bar{u}$, which is mapped onto $\Pi_{*} v_{n} \times u$ under $\Pi_{*}: H^{*}\left(; Z^{T}\right) \rightarrow$ $H^{*}\left(; Z_{2}\right)$. The result follows from (3.4.5).

Let $x \in H^{n}\left(X, A ; Z^{T}\right)$. If $n=0, x=0$. If $n=1$, the universal example is $X=K\left(Z^{T}, n\right), A=P_{\infty}$, and $x=v_{n}^{T}$, where $K\left(Z^{T}, n\right)$ is obtained as follows: ${ }^{1}$ Let $K(Z, n)$ be a topogical group, let $T(g, y)=$ $\left(g^{-1}, T y\right)$ for all $g \in K(Z, n)$ and $y \in S^{\infty}$. Let

1 Personal communication from C. T. C. Wall. 


$$
K\left(Z^{T}, n\right)=K(Z, n) \times S^{\infty} / T .
$$

We have inclusion and projection

$$
P_{\infty} \stackrel{i}{\longrightarrow} K\left(Z^{T}, n\right) \stackrel{p}{\longrightarrow} P_{\infty}
$$

where $i[y]=\left[{ }^{*}, y\right]$ and $p[g, y]=[y] ; P_{\infty}$ may thus be considered to be a subset of $K\left(Z^{T}, n\right)$, and its cohomology group is a direct summand. Then $v_{n}^{T} \in H^{n}\left(K\left(Z^{T}, n\right), P_{\infty} ; Z^{T}[u]\right)$ is the fundamental class.

$$
H^{n}\left(X, A ; Z_{2}\right) \cong Z_{2}
$$

is generated by $I_{*} v_{n}^{T} ; H^{n+1}\left(X, A ; Z_{2}\right) \cong Z_{2}$ generated by $I_{*} v_{n}^{T} \cup u$. Thus, by (3.4.3) and (3.4.4), $H^{n+1}(X, A ; Z) \cong Z_{2}$ generated by $v_{n}^{T} \cup \bar{u}$, and the result follows from (3.4.5).

(3.5.2). We summarize the results of (3.4.5) and (3.5.1) in the following commutative diagram with all rows exact:

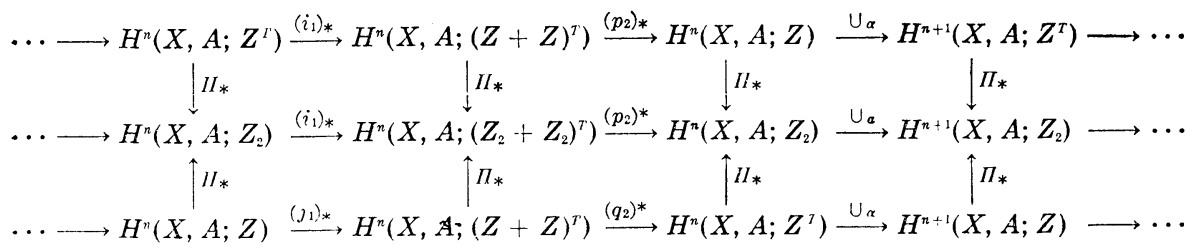

3.6. Applying the results of 3.4 and 3.5, we compute the cohomology of real projective space $P_{k}$, for $k \geqq 1$ :

$$
\begin{aligned}
H^{n}\left(P_{k} ; Z_{z}\right) \cong & \begin{cases}Z_{2}, & \text { generated by } u^{n}, \text { if } n \leqq k \\
0 \quad \text { if } n>k\end{cases} \\
H^{n}\left(P_{k} ; Z\right) \cong & \begin{array}{ll}
Z_{2}, & \text { generated by } \bar{u}^{n}, \text { if } n \\
& \text { even, } 0<n \leqq k \\
Z, & \text { generated by } 1, \text { if } n=0 \\
0, & \text { if } n \text { odd, } 0<n<k \\
Z, & \text { generated by } t\left(P_{k}\right), \text { the } \\
\text { top class, if } n=k \text { odd } \\
0, & \text { if } n>k .
\end{array}
\end{aligned}
$$

$$
H^{n}\left(P_{k} ; Z^{T}[u]\right) \cong \begin{cases}Z_{2}, & \text { generated by } \bar{u}^{n}, \text { if } n \text { odd } \\ 0<n \leqq k & \text { if } n \text { even, } 0<n<k \\ Z, & \text { generated by } t\left(P_{k}\right), \text { the top } \\ & \text { class, if } n=k \text { even } \\ 0, & \text { if } n>k .\end{cases}
$$


$(3.6 .4)$

$(3.6 .7)$

$$
\begin{aligned}
&\left.H^{n} P_{k}, * ; Z^{T}[u]\right) \cong\left\{\begin{array}{l}
0, \text { if } n=0 \\
Z, \text { generated by } \bar{u}, \text { if } n=1 \\
H^{n}\left(P_{k} ; Z^{T}[u]\right) \text { if } n>1
\end{array}\right. \\
& H^{n}\left(P_{k} ; Z_{2}+Z_{2}\right) \cong H^{n}\left(P_{k} ; Z_{2}\right) \oplus H^{n}\left(P_{k} ; Z_{2}\right) . \\
& H^{n}\left(P_{k} ; Z+Z\right) \cong H^{n}\left(P_{k} ; Z\right) \oplus H^{n}\left(P_{k} ; Z\right) .
\end{aligned}
$$

$(3.6 .8) \quad H^{n}\left(P_{k} ;\left(Z_{2}+Z_{2}\right)^{T}[u]\right) \cong\left\{\begin{aligned} Z_{2}, & \text { generated by }\left(i_{1}\right)_{*} 1 \\ & \text { if } n=0 \\ 0, & \text { if } 0<n<k \\ Z_{2}, & \text { generated by }\left(p_{2}\right)_{*}^{-1} u^{k} \\ & \left(=\Pi_{* \frac{1}{2}}\left(i_{1}\right)_{*} t\left(P_{k}\right)\right) \text { if } k \\ & \text { even, }=\Pi_{*}\left(j_{1}\right)_{*} t\left(P_{k}\right) \text { if } k \\ & \text { odd }) \text { if } n=k \\ 0, & \text { if } n>k .\end{aligned}\right.$

$Z$, generated by $\left(j_{1}\right)_{*} 1$, if $n=0$

0 , if $0<n<k$ $H^{n}\left(P_{k} ;(Z+Z)^{T}[u]\right) \cong \begin{cases}Z, & \text { generated by } \frac{1}{2}\left(i_{1}\right)_{*} t\left(P_{k}\right)= \\ & \left(q_{2}\right)_{*}^{-1} t\left(P_{k}\right) \text { if } n=k \text { is even }\end{cases}$

$Z$, generated by $\frac{1}{2}\left(j_{1}\right)_{*} t\left(P_{k}\right)=$ $\left(p_{2}\right)_{*}^{-1} t\left(P_{k}\right)$ if $n=k$ is odd

0 , if $n>k$

4. Evaluation of the differentials.

4.1. We need two remarks.

(4.1.1) If $Y_{1}$ and $Y_{2}$ are spaces, and $h: Y_{1} \rightarrow Y_{2}$ is a map, $h$ induces a map $\left(Y_{1}\right)_{n-1} \rightarrow\left(Y_{2}\right)_{n-1}$ and a sheaf homomorphism $\tilde{h}: \pi_{n}\left(Y_{1}, 1\right) \rightarrow$ $\pi_{n}\left(Y_{2}, h\right)$. If $k_{1}^{n+1}$ and $k_{2}^{n+1}$ are the $n^{\text {th }} k$-invariants of $Y_{1}$ and $Y_{2}$ respectively, $\widetilde{h}_{*} k_{1}^{n+1}=h^{*} k_{1}^{n+2} \in H^{n+1}\left(\left(Y_{1}\right)_{n-1} ; \pi_{n}\left(Y_{2}, h\right)\right)$.

(4.1.2) Let $X$ and $Y$ be spaces, $2 \leqq m<n$ integers such that $\pi_{k}(Y)=0$ for all $m<k<n$, and $f: X \rightarrow(Y)_{n}$ a map. If the $k$ invariant $k^{n+1}$ of $Y$ is based on the relation $\theta\left(1, k^{m+1}\right)=0$, where $\theta$ is a map cohomology operation and $1:(Y)_{m-1} \rightarrow(Y)_{m-1}$ is the identity map, then; for any

$$
x \in H^{m-1}\left(X ; \pi_{m}(Y, f)\right), d_{r}(x)=s^{-2} \theta\left(p_{m-1}^{n} f P, s^{2} x\right), r=n-m+1,
$$

where $P: X \times S^{2} \rightarrow X$ is projection,

$$
s^{2}: H^{*}\left(X, x_{0}\right) \rightarrow H^{*+2}\left(X \times S^{2}, X \times * \cup x_{0} \times S^{2}\right)
$$


is suspension and $p_{m-1}^{n}=p_{m} \cdots p_{n}:(Y)_{n} \rightarrow(Y)_{m-1}$.

Proof. Let $\left(S^{1}, *\right)$ be a circle, which we think of as the unit interval with end-points identified. Let $C: X \times S^{1} \rightarrow(Y)_{m}$ be the constant homotopy of $p_{m}^{n} f$ with itself. Now $p_{m}(C+s x)=p_{m} C$, where $C+s x$ is as defined in (1.2.2) and $d_{r}(x)=\delta^{n}(f, f ; C+s x)$ by (1.3). Finally, $s \hat{o}^{n}(f, f ; C+s x)=(C+s x)^{*} k^{n+1}=s^{-1} \theta\left(p_{m-1}^{n} f P, s^{2} x\right)$.

4.2. Kervaire [3, p. 162] gives us the following table of homotopy groups:

\begin{tabular}{ccccccccc}
\hline & $B O(1)$ & $B O(2)$ & $B O(3)$ & $B O(4)$ & $B O(5)$ & $B O(6)$ & $B O(n)$ & for $7 \leqq n \leqq \infty$ \\
\hline$\pi_{1}$ & $Z_{2}$ & $Z_{2}$ & $Z_{2}$ & $Z_{2}$ & $Z_{2}$ & $Z_{2}$ & $Z_{2}$ & \\
$\pi_{2}$ & 0 & $Z$ & $Z_{2}$ & $Z_{2}$ & $Z_{2}$ & $Z_{2}$ & $Z_{2}$ & \\
$\pi_{3}$ & 0 & 0 & 0 & 0 & 0 & 0 & 0 & \\
$\pi_{4}$ & 0 & 0 & $Z$ & $Z+Z$ & $Z$ & $Z$ & $Z$ & \\
$\pi_{5}$ & 0 & 0 & $Z_{2}$ & $Z_{2}+Z_{2}$ & $Z_{2}$ & 0 & 0 & \\
$\pi_{6}$ & 0 & 0 & $Z_{2}$ & $Z_{2}+Z_{2}$ & $Z_{2}$ & $Z$ & 0. & \\
\hline
\end{tabular}

Now $\pi_{1}(B O(n))=Z_{2}$ acts on $\pi_{k}(B O(n))$ for all $n \geqq 1, k \geqq 1$; this action is trivial if $\pi_{k}(B O(n))$ is stable, that is, $k<n$; because $B O$ is simple. For $n$ even, $Z_{2}$ acts nontrivially on $\pi_{n}(B O(n))$, because the first relative $k$-invariant of $B O(n) \rightarrow B O$ is

$$
k^{n+1}=\beta^{T}\left[w_{1}\right] w_{n} \in H^{n+1}\left(B O ; Z^{T}\left[w_{1}\right]\right) .
$$

(Because $\Pi_{*} k^{n+1}$, the reduction $\bmod 2$, must be $w_{n+1}$ ). $Z_{2}$ acts trivially on $\pi_{4}(B O(3))$ because if acts trivially on $\pi_{4}(B O)$ and the map $Z \cong$ $\pi_{4}(B O(3)) \rightarrow \pi_{4}(B O) \cong Z$ is just multiplication by 2 . Since $Z_{2}$ can only act trivially on $Z_{2}$, we need only now examine the action on $\pi_{4}(B O(4))$ for $k=4,5,6$.

Proposition (4.2.1). We may choose generators $x$ and $y$ of $\pi_{4}(B O(4))$ such that $T(x)=-x, T(y)=x+y$, and the maps

$$
i_{4}^{3}: \pi_{4}(B O(3)) \longrightarrow \pi_{4}(B O(4)) \text { and } i_{4}^{4}: \pi_{4}(B O(4)) \longrightarrow \pi_{4}(B O(5))
$$

have the properties $i_{4}^{3}(1)=x+2 y, i_{4}^{4}(x)=0$ and $i_{4}^{4}(y)=1$.

Proof. We know that $i_{4}^{4}$ is onto. Choose $x$ to be a generator of $\operatorname{Ker} i_{4}^{4}$, and pick $a$ such that $i_{4}^{4} a=1$. Now $2 a-i_{4}^{3}(1) \in \operatorname{Ker} i_{4}^{4}$, since $i_{4}^{4} i_{4}^{3}=2$. So $2 a-i_{4}^{3}(1)$ is a multiple of $x$. It can't be an even multiple, because then $i_{4}^{3}(1)$ would be divisible by 2 , and $i_{4}^{3} \pi_{4}(B O(3))$ is a direct summand of $\pi_{4}(B O(4))$. So for some $k, 2 a-i_{4}^{3}(1)=(2 k-1) x$. Let $y=$ $a-k x$; then $i_{4}^{3}(1)=x+2 y, i_{4}^{4}(x)=0$, and $i_{4}^{4}(y)=1$. Now $T(x) \in \operatorname{Ker} i_{4}^{4}$, so $T(x)$ must be $-x$. $T(x+2 y)=x+2 y$ so $T(y)=\frac{1}{2}(x+2 y-T x)=$ $x+y$. We are done. 
We represent $\pi_{4}(B O(4))$ as ordered pairs of integers, where $(p, q)$ represents $p x+q y$.

Proposition (4.2.2). $\pi_{5}(B O(4))$ and $\pi_{6}(B O(4))$ may be represented as ordered pairs of elements of $Z_{2}$, such that $i_{5}^{3}(x)=i_{6}^{3}(x)=(x, 0)$, $i_{5}^{4}(x, y)=i_{6}^{1}(x, y)=y$, and $T(x, y)=(x+y, y)$ for all $x, y \in Z_{2}$.

Proof. $\pi_{5}(B O(n))$ and $\pi_{6}(B O(n))$ are the images, under $\eta$ and $\eta^{2}$ respectively, of $\pi_{4}(B O(n))$, for $n=3,4$, or 5. Apply (4.2.1).

REMARK (4.2.3). There are two possible choices of $x$ in (4.2.1) we retroactively make that choice such that the image of $\pi_{5}(B U(2)) \cong Z_{2}$, under the classifying map of the reallification $B U(2) \rightarrow B O(4)$, is generated by $(0,1) \in \pi_{5}(B O(4))$.

4.3. We need to describe $k$-invariants for $B O(n)$.

(4.3.1) For all $n, k^{3}$ of $B O(n)$ is zero, since the projection

$$
P_{1}: B O(n) \longrightarrow(B O(n))_{1}=K\left(Z_{2}, 1\right)=B O(1)
$$

has a lifting, namely, the map induced by the inclusion of $O(1)$ in $O(n)$. Also $k^{4}=0$, since $\pi_{3}(B O(n))=0$.

(4.3.2) For $B O(3), k^{5}= \pm \beta_{4} \mathfrak{s} w_{2}$, where $\beta_{4}$ is the Bockstein of $Z \rightarrow$ $Z \rightarrow Z_{4}$ and $\mathfrak{P}: H^{2}\left(; Z_{2}\right) \rightarrow H^{4}\left(; Z_{4}\right)$ is the Pontrjagin square [2], and $k^{6}$ is based on the relation $S_{q}^{2} \Pi_{*} k^{5}+w_{2} \cup \Pi_{*} k^{5}=0$.

(4.3.3) For $B O(5), k^{5}=2 \beta_{4} \Re w_{2}=\beta w_{2}^{2}$ (see [4]), and $k^{6}=w_{6}$, based on the relation $S_{q}^{2} I I_{*} k^{5}+w_{2} \cup \Pi_{*} k^{5}=0$.

(4.3.4) Using (4.3.2), (4.3.3), we get that for $B O(4), k^{5}=\iota \beta_{4} \Re w_{2}$, where $\iota: H^{*}(; Z) \rightarrow H^{*}\left(;(Z+Z)^{T}\right)$ is $\left(j_{1}\right)_{*}$ as described in (3.5.2), and $k^{5}$ is of order 4 and generates $H^{5}\left((B O(4))_{4} ;(Z+Z)^{T}\left[w_{1}\right]\right)$. Also, $k^{6}$ is based on the relation $S_{q}^{2} \Pi_{*} k^{5}+w_{2} \cup \Pi_{*} k^{5}$, where

$$
S_{q}^{2}: H^{*}\left(;\left(Z_{2}+Z_{2}\right)^{T}[a]\right) \longrightarrow H^{*+2}\left(;\left(Z_{2}+Z_{2}\right)^{T}[a]\right)
$$

is that unique operation which is ordinary $S_{q}^{2}$ on each factor when $a=0$, and $w_{2} \cup$ is as described in (3.5).

(4.3.5) For $B O(6), k^{5}=2 \beta_{4} \Re w_{2}=\beta w_{2}^{2}$, and $k^{7}=\beta^{T}\left[w_{1}\right] w_{6}$, based on the relation $\beta^{T}\left(S_{q}^{2} \Pi_{*} k^{5}+w_{2} \cup \Pi_{*} k^{5}\right)=0$.

4.4. Using (4.1.1) and (4.1.2) we can now evaluate some differentials $d_{r}=d_{r}^{f}$ for a map $f: X \rightarrow(Y)_{k}$.

(4.4.1) If $Y=B O(1)$ or $B O(2), d_{r}=0$.

(4.4.2) If $Y=B O(3)$ and $k<4, d_{r}=0$. If $k=4, d_{2}=0$ : by (4.1.2), $d_{3}(x)=\beta\left(x^{3}+x \cup f^{*} w_{2}\right) \in H^{4}(X ; Z)$ for all $x \in H^{1}\left(X ; Z_{2}\right)$. This was also known to Dold and Whitney [2]. If 


$$
k=5, d_{2}(x)=S_{q}^{2} \Pi_{*} x+f^{*} w_{2} \cup \Pi_{*} x \in H^{5}\left(X ; Z_{2}\right),
$$

for all $x \in H^{3}(X ; Z)$ by (4.1.2); $d_{3}=0$, and $d_{4}$ requires special computation. (4.1.2),

(4.4.3) If $Y=B O(4)$ and $k<4, d_{r}=0$. If $k=4, d_{2}=0$; and by

$$
d_{3}(x)=\iota \beta\left(x^{3}+x \cup f^{*} w_{2}\right) \in H^{4}\left(X ;(Z+Z)^{T}\left[f^{*} w_{1}\right]\right)
$$

for all $x \in H^{1}\left(X ; Z_{2}\right)$; if

$$
k=5, d_{2}(x)=S_{q}^{2} \Pi_{*} x+f^{*} w_{2} \cup \Pi_{*} x \in H^{5}\left(X ;\left(Z_{2}+Z_{2}\right)^{T}\left[f^{*} w_{1}\right]\right)
$$

for all $x \in H^{3}\left(X ;(Z+Z)^{T}\left[f^{*} w_{1}\right]\right)$ by (4.1.2), $d_{3}=0$, and $d_{4}$ must be computed specially.

$$
\begin{aligned}
& \text { (4.4.4) If } Y=B O(5) \text { and } k<5, d_{r}=0 \text {. If } \\
& k=5, d_{2}(x)=S_{q}^{2} I_{*} x+f^{*} w_{2} \cup \Pi_{*} x \in H^{5}\left(X ; Z_{2}\right)
\end{aligned}
$$

for all $x \in H^{3}(X ; Z), d_{3}=0$, and

$$
\begin{aligned}
d_{4}(x)= & x^{5}+f^{*} w_{1} \cup x^{4}+f^{*} w_{2} \cup x^{3}+f^{*} w_{3} \cup x^{2} \\
& +f^{*} w_{4} \cup x+\operatorname{Im} d_{2} \in E_{4}^{5,5}=H^{5}\left(X ; Z_{2}\right) / \operatorname{Im} d_{2}
\end{aligned}
$$

for all $x \in H^{1}\left(X ; Z_{2}\right)$.

Proof. We have a map $S: \Sigma K(Z, 1)-B S O$, such that $S^{*} w_{i+1}=s u^{i}$ for all $i \geqq 1$, where $u$ is the fundamental class. Now $(B O(5))_{4}=(B O)_{4}$ has the same homotopy as $B O$ up through dimension 7 , so we identify $H^{k}\left((B O(5))_{4}\right.$ with $H^{k}(B O)$ for $0 \leqq k \leqq 7$. Let $h: \Sigma K\left(Z_{2}, 1\right)-(B O(5))_{4}$ be given by the commutative diagram:

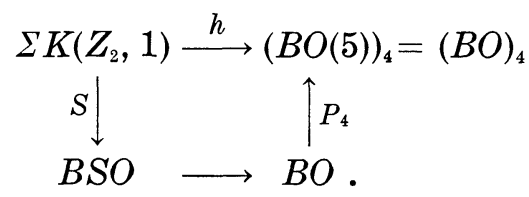

$(B O(5))_{4}$ has an $H$-space structure $\mu:(B O(5))_{4} \times(B O(5))_{4} \rightarrow(B O(5))_{4}$ and $\mu^{*} w_{6}=\sum_{i=0}^{6} w_{i} \times w_{6-i}$. Let $Q X$ be the space obtained from $X \times S^{1}$ by collapsing $x_{0} \times S^{1}$; let $J: Q X \rightarrow \Sigma X$ be the map which collapses $X \times{ }^{*}$, and let $p_{1}: Q X \rightarrow X$ be projection onto the first factor. For any $x \in\left(H^{*} X\right)$, let $q x=p_{1}^{*} x$ and let $Q x=J^{*} s x$, both in $H^{*}(Q X)$. We showed in [4, 5.1] that $q a \cup q b=q(a \cup b), q a \cup Q b=Q(a \cup b)$, and $Q a \cup Q b=0$ for all $a, b \in H^{*}(X)$. Let $C: X \rightarrow K\left(Z_{2}, 1\right)$ be a classifying map for a given $x \in H^{1}\left(X ; Z_{2}\right)$, and let $F: Q X \rightarrow(B O(5))_{4}$ be a map, which represents a homotopy of $p_{5} f$ with itself, defined by composing the following maps: 


$$
\begin{aligned}
& Q X \stackrel{\Delta}{\longrightarrow} Q X \times Q X \stackrel{J \times p_{1}}{\longrightarrow} \Sigma X \times X \stackrel{\Sigma C \times p_{5} f}{\longrightarrow} \Sigma K\left(Z_{2}, 1\right) \times(B O(5))_{4} \\
& \stackrel{h \times 1}{\longrightarrow}(B O(5))_{4} \times(B O(5))_{4} \stackrel{\mu}{\longrightarrow}(B O(5))_{4} .
\end{aligned}
$$

By (1.3), $d_{4}(x)$ contains $\delta^{5}(f, f ; F)$. Now routine computation shows that $f^{*} w_{6}=Q\left(x^{5}+x^{4} f^{*} w_{1}+x^{3} f^{*} w_{2}+x^{2} f^{*} w_{3}+x f^{*} w_{4}\right)$, and the result follows from [4, 5.2].

(4.4.5) If $Y=B O(6)$ and $k<6, d_{r}=0$. If $k=6, d_{2}=0$ and $d_{3}(x)=\beta^{T}\left(S_{q}^{2} \Pi_{*} x+f^{*} w_{2} \cup \Pi_{*} x\right) \in H^{6}\left(X ; Z^{T}\left[f^{*} w_{1}\right]\right)$ for all $x \in H^{3}(X ; Z)$; $d_{4}=0$ and

$$
\begin{aligned}
d_{5}(x)= & \beta^{T}\left(x^{5}+x^{4} f^{*} w_{1}+x^{3} f^{*} w_{2}+x^{2} f^{*} w_{3}+x f^{*} w_{4}\right) \\
& +\operatorname{Im} d_{2} \in E_{5}^{6,6}=H^{6}\left(X ; Z^{T}\left[f^{*} w_{1}\right]\right) / \operatorname{Im} d_{3}
\end{aligned}
$$

for all $x \in H^{1}\left(X ; Z_{2}\right)$.

Proof. same as (4.4.4).

4.5. We are now ready to classify real vector bundles over $P_{k}$, for $k \leqq 5$.

Definition (4.5.1). A locally oriented real $n$-dimensional vector bundle over a space $X$ shall be a b.p.p. homotopy class of maps from $X$ to $B O(n)$. If $f: X \rightarrow B O(n)$ represents a locally oriented v.b. $\xi$, let $\sim \xi$, or $\xi$ conjugate, be that locally oriented v.b. given by a map $g: X \rightarrow B O(n)$ which is connected to $f$ via a free homotopy which sends the base-point of $X$ around a nontrivial loop of $B O(n)$. Obviously $\sim \xi \cong \xi$, and conjugate classes of locally oriented vector bundles correspond to equivalence classes of vector bundles.

TABLE (4.5.2). For $k \geqq 1$, let $h: P_{k} \rightarrow B O(1)$ be the canonical line bundle. Let " $\oplus$ " denote Whitney sum. We give a complete list of all locally oriented real $n$-dimensional vector bundles over $P_{k}$, each $n$ and $k$; all bundles are self-conjugate unless otherwise specified.

Let $G$ denote $\left(q_{1}\right)_{*}^{-1} t\left(P_{4}\right)=\frac{1}{2}\left(i_{1}\right)_{*} t\left(P_{4}\right)$ which generates

\begin{tabular}{|c|c|c|c|c|}
\hline \multicolumn{2}{|c|}{ Over $P_{1}$} & \multicolumn{3}{|c|}{ Over $P_{2}$} \\
\hline 1 & 2 & 1 & 2 & 3 \\
\hline$h$ & $h \oplus 1$ & $h$ & $\begin{aligned} T_{p}= & (h \oplus 1)+p t\left(P_{2}\right), \text { for all } p \in Z ; \\
& \text { stable class } h+1 \text { if } p \text { even, } \\
& 3 h-1 \text { if } p \text { odd; } \sim T_{p}=T_{-p} . \\
2 h= & 2+\bar{u}^{2}\end{aligned}$ & $\begin{array}{l}h \oplus 2 \\
2 h \oplus 1=3+u^{2} \\
3 h=(h \oplus 2)+u^{2}\end{array}$ \\
\hline
\end{tabular}

$$
H^{4}\left(P_{4} ;(Z+Z)^{T}[u]\right) \text {. }
$$

Also $\left(p_{2}^{*}\right)^{-1} u^{5}$ generates $H^{5}\left(P_{5} ;\left(Z_{2}+Z_{2}\right)^{T}[u]\right)$. Locally oriented real $n$-dimensional vector bundles over $P_{k}$, for $n-1 \leqq k \leqq 5$ : 


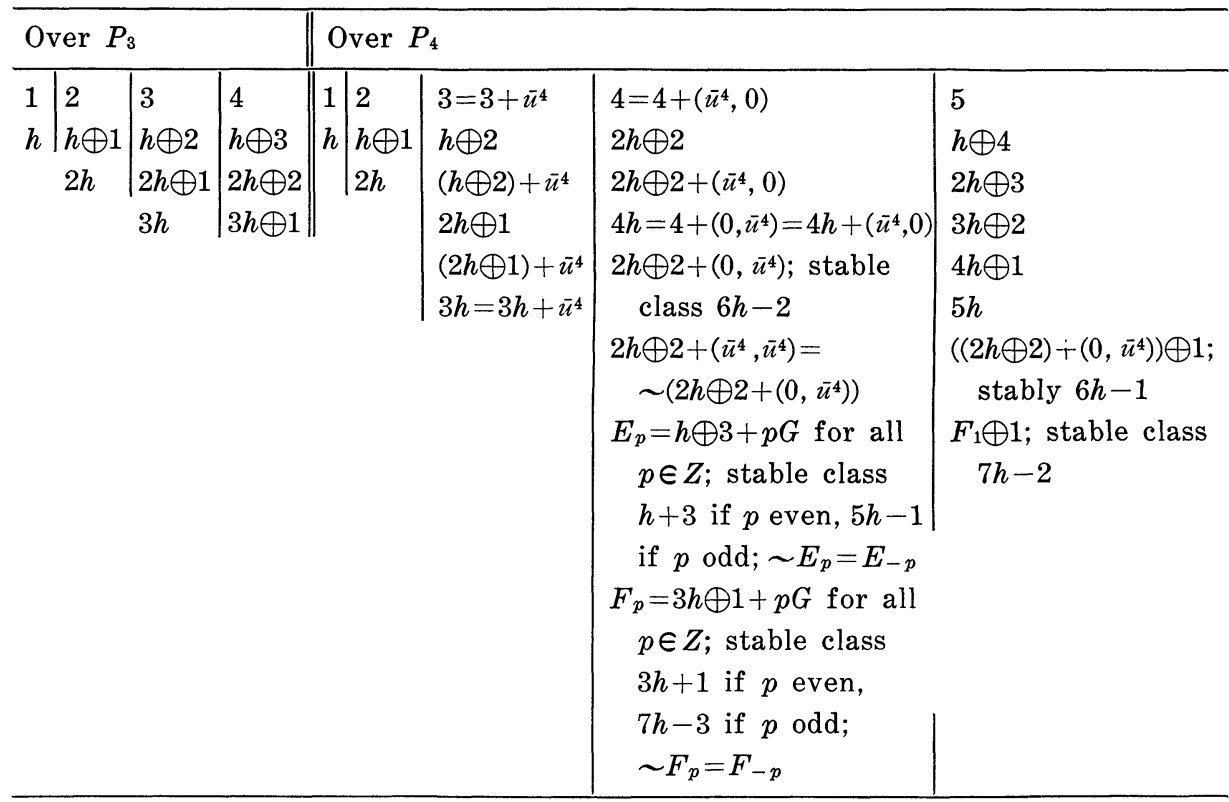

\section{Over $P_{5}$}

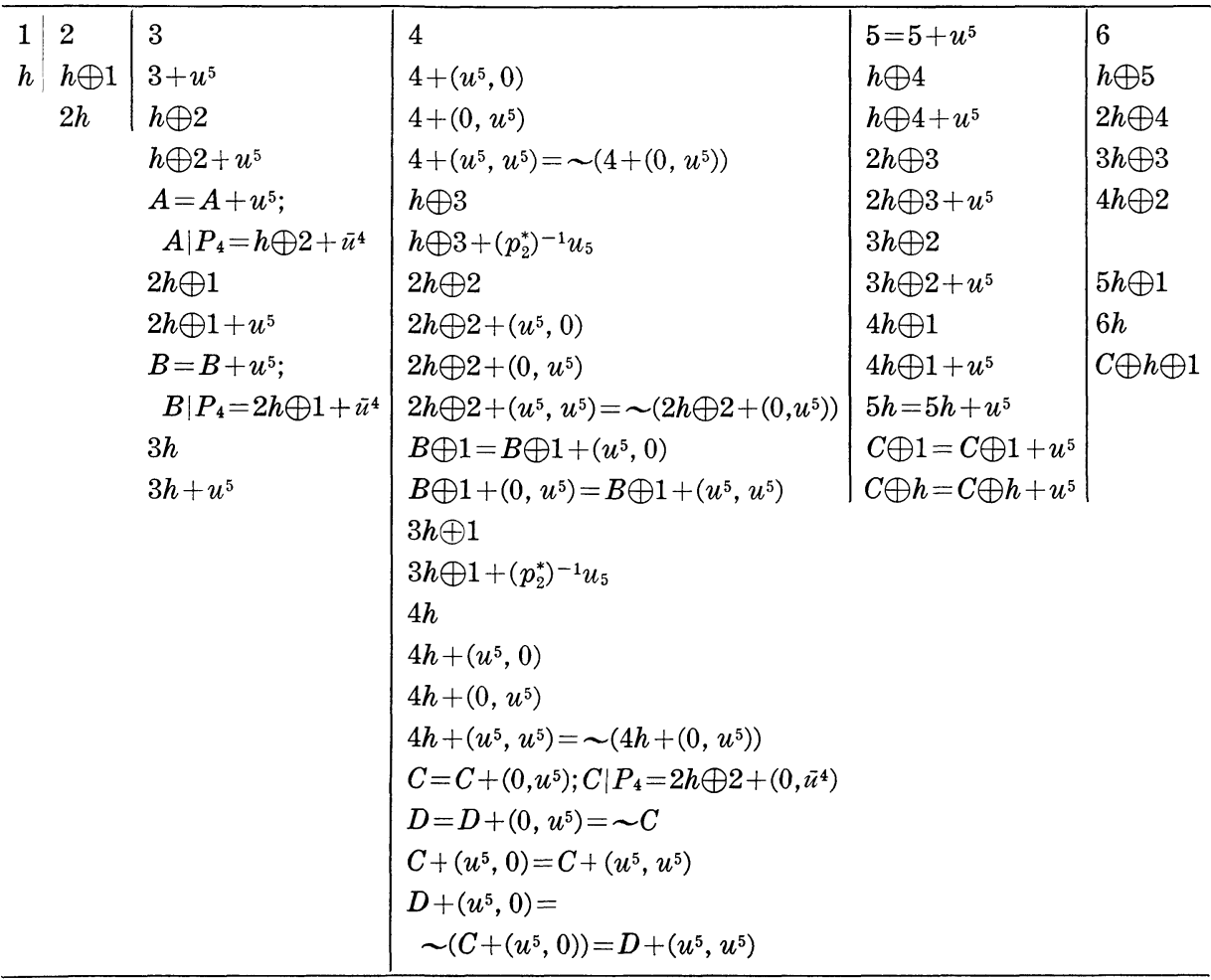


4.6. Similarly, we can classify all complex vector bundles over $P_{k}$, for $k \leqq 5$. We give a table of homotopy groups:

\begin{tabular}{ccccc}
\hline & $B U(1)$ & $B U(2)$ & $B U(n)$ & for $3 \leqq n \leqq \infty$ \\
\hline$\pi_{1}$ & 0 & 0 & 0 & \\
$\pi_{2}$ & $Z$ & $Z$ & $Z$ & \\
$\pi_{3}$ & 0 & 0 & 0 & \\
$\pi_{4}$ & $Z$ & $Z$ & $Z$ & \\
$\pi_{5}$ & 0 & $Z_{2}$ & 0 & \\
\hline
\end{tabular}

The only nonzero $k$-invariant in this range is $k^{6}$ of $B U(2)$, which is $\Pi_{*}\left(c_{1} c_{2}\right)+S_{q}^{2} \Pi_{*} c_{2}$, where $c_{i} \in H^{2 i}(B U(2) ; Z)$ are the Chern classes. We thus have:

Remark (4.6.1). For any space $X$, all complex line bundles over $X$ correspond to $H^{2}(X ; Z)$.

REMARK (4.6.2). For any space $X$ of dimension $\leqq 5$, all complex $n$-bundles, for $n \geqq 3$, over $X$ correspond to $K U(X)$, satisfying the exact sequence $0 \rightarrow H^{4}(X ; Z) \rightarrow K U(X) \rightarrow H^{2}(X ; Z) \rightarrow 0$.

REMARK (4.6.3). If $f: X \rightarrow(B U(2))_{5}$ is a map, then

$$
d_{2}(x)=\Pi_{*}\left(c_{1} x\right)+S_{q}^{2} \Pi_{*} x \in H^{5}\left(X ; Z_{2}\right)
$$

for all $x \in H^{3}(X ; Z) ; d_{3}=0 ; d_{4}(x)=\Pi_{*}\left(f^{*} c_{2} \cup x\right)+\operatorname{Im} d_{2}$ for all

$$
x \in H^{1}(X ; Z) \text {. }
$$

Proof. Let $S: S^{2}=\Sigma K(Z, 1) \rightarrow B U$ be the generator of $\pi_{2}(B U)$; then $S^{*} c_{1}=\sigma$, the fundamental class of $S^{2}$, and $S^{*} c_{2}=0$. The result follows just as in (4.4.4).

TABLE (4.6.4). We summarize complex $n$-bundles over $P_{k}, 2 n-$ $1 \leqq k \leqq 5$. The reallification is given in square brackets.

\begin{tabular}{ll|ll||ll|ll}
\hline \multicolumn{3}{c}{ Over $P_{2}$} & \multicolumn{5}{c}{ Over $P_{3}$} \\
\hline 1 & {$[2]$} & 2 & {$[4]$} & 1 & {$[4]$} & 2 & {$[4]$} \\
$H$ & {$[2 h]$} & $H \oplus 1=2+u^{2}$ & {$[2 h \oplus 2]$} & $H$ & {$[2 h]$} & $H \oplus 1$ & {$[2 h \oplus 2]$} \\
\hline
\end{tabular}

Over $P_{4}$

\begin{tabular}{ll|ll|ll}
1 & {$[2]$} & 2 & {$[4]$} & 3 & {$[6]$} \\
$H$ & {$[2 h]$} & $H \oplus 1$ & {$[2 h \oplus 2]$} & $H \oplus 2$ & {$[2 h \oplus 4]$} \\
& & $2 H=2+\bar{u}^{4}$ & {$[4 h]$} & $2 H \oplus 1=3+\bar{\iota}^{4}$ & {$[4 h \oplus 2]$} \\
& $H \oplus 1+\bar{u}^{4}$ & {$\left[2 h \oplus 2+\left(\bar{u}^{4}, 0\right)\right]$} & $3 H=H \oplus 2+\bar{u}^{4}$ & {$[6 h]$}
\end{tabular}

Stable class $3 H-1$ 


\begin{tabular}{|c|c|c|c|}
\hline \multicolumn{4}{|c|}{ Over $P_{5}$} \\
\hline & [2] & 2 & {$[4]$} \\
\hline \multirow{7}{*}{\multicolumn{2}{|c|}{$[2 h]$}} & $2+u^{5}$ & {$\left[4+\left(0, u^{5}\right)\right]$} \\
\hline & & $H \oplus 1$ & {$[2 h \oplus 2]$} \\
\hline & & $H \oplus 1 \oplus u^{5}$ & {$\left[2 h \oplus 2+\left(0, u^{5}\right)\right]$} \\
\hline & & $2 H$ & {$[4 h]$} \\
\hline & & $2 H+u^{5}$ & {$\left[4 h+\left(0, u^{5}\right)\right]$} \\
\hline & & $C$ & {$[C]$} \\
\hline & & $C+u^{5}$ & {$[C]$} \\
\hline
\end{tabular}

4.7. We give a few representative examples of evaluating those difficult differentials. Is $f: P_{5} \rightarrow\left(B O_{4}\right)_{5}$ is a map representing a 4-plane bundle $\xi$, then $d_{4}^{f}(u)$ is defined if and only if

$$
d_{2}^{f}(u)=\left(j_{1}\right)_{*} \beta\left(u^{3}+u f^{*} w_{2}\right)=0 \in H^{4}\left(P_{5} ;(Z+Z)^{T}\left[f^{*} w_{1}\right]\right) .
$$

If $d_{2}(u)=0$, then $d_{4}^{f}(u)=0$ if and only if there is a map $F: Q P_{5} \rightarrow$ $\left(B O_{4}\right)_{5}$ which represents a homotopy of $f$ with itself, such that $F^{*} w_{2}=q f^{*} w_{2}+Q u$, where $Q X$ is as given in [4;5].

ExAmPLE (4.7.1). If $\xi=4$ or $4 h$, then $f^{*} w_{2}=0$, so $d_{2}(u)=\left(\bar{u}^{4}, 0\right)$ and $d_{4}(u)$ is not defined. Thus $4,4+\left(u^{5}, 0\right), 4+\left(0, u^{5}\right)$, and $4+\left(u^{5}, u^{5}\right)$ are all distinct oriented vector bundles.

EXAMPLE (4.7.2). If $\xi=2 h \oplus 2$, then $f^{*} w_{2}=u^{2}$, so $d_{2}(u)=0$.

Let $\eta_{1}$ be that line bundle over $Q P_{5}$ such that $w_{1}\left(\eta_{1}\right)=q u$; now 2-plane bundles over a space $X$ with $w_{1}=x$ are classified by $H^{2}\left(X ; Z^{T}[x]\right)$; let $\eta_{2}$ be that 2-plane bundle over $Q P_{5}$ with $\mathrm{w}_{1}\left(\eta_{2}\right)=q u$ classified by $Q \bar{u}$. Then $w_{2}\left(\eta_{2}\right)=Q u$. Let $c: Q P_{5} \rightarrow B O(4)$ be the classifying map of $\eta_{1} \oplus \eta_{2} \oplus 1 ; c^{*} w_{2}=q u^{2}+Q u$ and $\left(\eta_{1} \oplus \eta_{2} \oplus 1\right) \mid P_{5}=2 h \oplus 2$. Thus $F$, the projection of $c$ onto $(B O(4))_{5}$, and $d_{4}^{f}(u)=0$.

EXAMPLe (4.7.3). If $\xi=C$, then $f^{*} w_{2}=u^{2}$, so $d_{2}^{f}(u)=0$, and $d_{4}^{f}(u)$ is defined. Now $p_{5} C=p_{5}(2 h \oplus 2)+\left(0, \bar{u}^{4}\right)$,

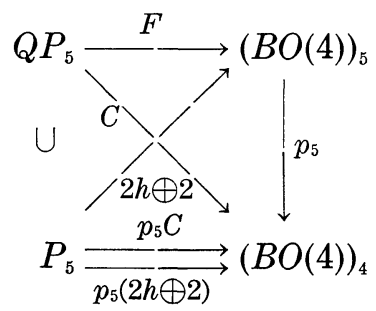

and so $d_{4}(u)=0$ if and only if we can lift the map 


$$
p_{5} F+q\left(0, \bar{u}^{4}\right): Q p_{5} \longrightarrow\left((B O(4))_{4}\right.
$$

to $(B O(4))_{5}$, where $F$ is the map given in (4.7.2). Now the $k$-invariant $k^{6}$ is based on the relation $S_{q}^{2} \Pi_{*} k^{5}+w_{2} \cup \Pi_{*} k^{5}=0$, and $\left(p_{5} F\right)^{*} k^{6}=0$, so $\left(p_{5} F+a\right)^{*} k^{6}=S_{q}^{2} \Pi_{*} a+\left(p_{5} F\right)^{*} w_{2} \cup \Pi_{*} a$ which, when $a=q\left(0, \bar{u}^{4}\right)$, equals $S_{q}^{2} q\left(0, u^{4}\right)+\left(q u^{2}+Q u\right) \cup q\left(0, u^{4}\right)=Q\left(0, u^{5}\right)$. So, by [4; 5.2], $d_{4}(u)=$ $\left(0, u^{5}\right)$. Thus $C+\left(0, u^{5}\right)=C$, but $C+\left(u^{5}, 0\right)$ is different. We also have that there are two complex structures on $C$, because since $C$ is the reallification of the complex bundle $C, C=C+\left(0, u^{5}\right)$ is the reallification of $C+u^{5}$.

4.8. We would like to know how vector bundles behave under tensor products. If $L$ is any line bundle over any space, $L \otimes L=1$. Furthermore:

REMARK (4.8.1). If $\eta_{1}$ and $\eta_{2}$ are locally oriented real $n$-plane bundles over a space $X$, which agree on $X^{k-1}$, and if $\xi$ is a locally oriented real $m$-plane bundle over $X$, then $i_{*} \delta^{k}\left(\eta_{1}, \eta_{2}\right)=\delta^{k}\left(\eta_{1} \oplus \xi, \eta_{1} \oplus \xi\right)$ and $j_{*} \delta^{k}\left(\eta_{1}, \eta_{2}\right)=d^{k}\left(\eta_{1} \otimes \xi, \eta_{2} \otimes \xi\right)$, where $i: B O(n) \rightarrow B O(n+m)$ and $j: B O(n) \subset B O(n m)$ are the maps induced by the inclusion of $O(n)$ in $O(n+m)$ and $O(n m)$. Similarly for complex vector bundles.

REMARK (4.8.2). If $\xi$ is an oriented real vector bundle which has a complex structure, and if $\eta$ is any other locally oriented real vector bundle, then $\xi \otimes \eta$ also has a complex structure.

Proof. Let $C(\eta)$ be the complexification of $\eta$, and let $\xi^{\prime}$ be a complex bundle whose reallification is $\xi$. Then we can see routinely that the reallification of $\xi^{\prime} \otimes C(\eta)$ is $\xi \otimes \eta$.

With the above information, we can almost completely determine the action of " $\oplus$ " and " $\otimes$ " on all locally oriented real vector bundles over $P_{k}, k \leqq 5$. For example,

$$
\begin{aligned}
A \otimes h & =B, C \otimes h=C, 4 \otimes h=4 h,\left(4+\left(0, u^{5}\right)\right) \otimes h=4 h+\left(0, u^{5}\right), \\
T_{p} \otimes h & =T_{p}, E_{p} \otimes h=F_{p},\left(4 h+\left(u^{5}, u^{5}\right)\right) \oplus 1=4 h \oplus 1+u^{5} .
\end{aligned}
$$

The only unsolved questions are whether $A \oplus h=B \oplus 1$; it is also possible that $A \oplus h=B \oplus 1+\left(0, u^{5}\right)$; and whether $B \oplus 2$ equals $2 h \oplus 3$ or $2 h \oplus 3+u^{5}$.

\section{BIBLIOGRAPHY}

1. G. E. Bredon, Sheaf theory, McGraw Hill, 1967.

2. A. Dold \& H. Whitney, Classification of oriented sphere bundles over a 4-complex, Ann. of Math. 69 (1959). 
3. M. A. Kervaire, Some nonstable homotopy groups of Lie groups, Illinois J. Math. 4 (1960), 161-169.

4. L. L. Larmore, Map-cohomology operations and enumeration of vector bundles, J. Math. Mech. 17 (1967), 199-208.

5. H. Samelson, $A$ note on the bokstein operator, Proc. Amer. Math. Soc. 15 (1964), 450-453.

Received January 20, 1968, and in revised form January 27, 1969. This research was supported by N.S.F. grant GP-6560.

Occidental College and

University of Illinois at Chicago Circle 



\title{
PACIFIC JOURNAL OF MATHEMATICS
}

\author{
EDITORS
}

H. ROYDEN

Stanford University

Stanford, California

R. R. PhelPS

University of Washington

Seattle, Washington 98105
J. DUGUNDJI

Department of Mathematics

University of Southern California

Los Angeles, California 90007

RICHARD ARENS

University of California

Los Angeles, California 90024

\section{ASSOCIATE EDITORS}
E. F. BECKENBACH
B. H. NeumanN
F. WOLF
K. YoSHIDA

\section{SUPPORTING INSTITUTIONS}

\author{
UNIVERSITY OF BRITISH COLUMBIA \\ CALIFORNIA INSTITUTE OF TECHNOLOGY \\ UNIVERSITY OF CALIFORNIA \\ MONTANA STATE UNIVERSITY \\ UNIVERSITY OF NEVADA \\ NEW MEXICO STATE UNIVERSITY \\ OREGON STATE UNIVERSITY \\ UNIVERSITY OF OREGON \\ OSAKA UNIVERSITY \\ UNIVERSITY OF SOUTHERN CALIFORNIA
}

\author{
STANFORD UNIVERSITY \\ UNIVERSITY OF TOKYO \\ UNIVERSITY OF UTAH \\ WASHINGTON STATE UNIVERSITY \\ UNIVERSITY OF WASHINGTON \\ $\stackrel{*}{*} \stackrel{*}{*}{ }^{*}{ }^{*}$ \\ CHEVRON RESEARCH CORPORATION \\ TRW SYSTEMS \\ NAVAL WEAPONS CENTER
}

The Supporting Institutions listed above contribute to the cost of publication of this Journal, but they are not owners or publishers and have no responsibility for its content or policies.

Mathematical papers intended for publication in the Pacific Journal of Mathematics should be in typed form or offset-reproduced, double spaced with large margins. Underline Greek letters in red, German in green, and script in blue. The first paragraph or two must be capable of being used separately as a synopsis of the entire paper. It should not contain references to the bibliography. Manuscripts, in duplicate if possible, may be sent to any one of the four editors. Please classify according to the scheme of Math. Rev. 36, 1539-1546. All other communications to the editors should be addressed to the managing editor, Richard Arens, University of California, Los Angeles, California, 90024.

50 reprints are provided free for each article; additional copies may be obtained at cost in multiples of 50 .

The Pacific Journal of Mathematics is published monthly. Effective with Volume 16 the price per volume (3 numbers) is $\$ 8.00$; single issues, $\$ 3.00$. Special price for current issues to individual faculty members of supporting institutions and to individual members of the American Mathematical Society: $\$ 4.00$ per volume; single issues $\$ 1.50$. Back numbers are available.

Subscriptions, orders for back numbers, and changes of address should be sent to Pacific Journal of Mathematics, 103 Highland Boulevard, Berkeley, California, 94708.

PUBLISHED BY PACIFIC JOURNAL OF MATHEMATICS, A NON-PROFIT CORPORATION

Printed at Kokusai Bunken Insatsusha (International Academic Printing Co., Ltd.), 7-17, Fujimi 2-chome, Chiyoda-ku, Tokyo, Japan. 


\section{Pacific Journal of Mathematics \\ Vol. 30, No. $2 \quad$ October, 1969}

Gregory Frank Bachelis, Homomorphisms of annihilator Banach algebras.

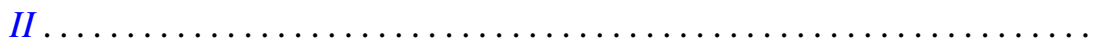

Leon Bernstein and Helmut Hasse, An explicit formula for the units of an algebraic number field of degree $n \geq 2 \ldots \ldots \ldots \ldots \ldots \ldots \ldots . \ldots 29$

David W. Boyd, Best constants in a class of integral inequalities ........ 367

Paul F. Conrad and John Dauns, An embedding theorem for lattice-ordered

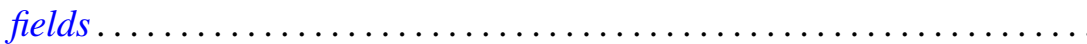

H. P. Dikshit, Summability of Fourier series by triangular matrix

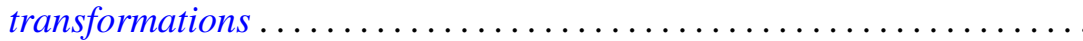

Dragomir Z. Djokovic, Linear transformations of tensor products preserving a fixed rank............................. 411

John J. F. Fournier, Extensions of a Fourier multiplier theorem of Paley . . . 415 Robert Paul Kopp, A subcollection of algebras in a collection of Banach

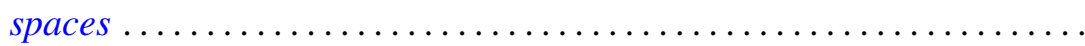

Lawrence Louis Larmore, Twisted cohomology and enumeration of vector bundles ...................................... 437

William Grenfell Leavitt and Yu-Lee Lee, A radical coinciding with the lower radical in associative and alternative rings .................

Samuel Merrill and Nand Lal, Characterization of certain invariant subspaces of $H^{p}$ and $L^{p}$ spaces derived from logmodular

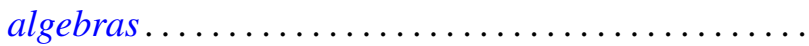

Sam Bernard Nadler, Jr., Multi-valued contraction mappings ....

T. V. Panchapagesan, Semi-groups of scalar type operators in Banach spaces ....................................

J. W. Spellmann, Concerning the infinite differentiability of semigroup motions

H. M. (Hari Mohan) Srivastava, A note on certain dual series equations involving Laguerre polynomials.

Ernest Lester Stitzinger, A nonimbedding theorem of associative algebras................................

J. Jerry Uhl, Jr., Martingales of vector valued set functions ...

John Mays Worrell Jr., On continuous mappings of metacompact $\check{C} e c h$

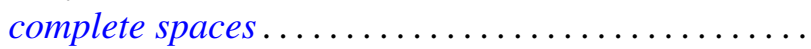

